\title{
Immune Centroids Oversampling Method for Binary Classification
}

\author{
Xusheng Ai, ${ }^{1}$ Jian Wu, ${ }^{1}$ Victor S. Sheng, ${ }^{2}$ Pengpeng Zhao, ${ }^{1}$ and Zhiming Cui ${ }^{1}$ \\ ${ }^{1}$ The Institute of Information Processing and Application, Soochow University, Suzhou 215006, China \\ ${ }^{2}$ Department of Computer Science, University of Central Arkansas, Conway, AR 72035, USA \\ Correspondence should be addressed to Jian Wu; jianwu@suda.edu.cn
}

Received 11 November 2014; Accepted 14 February 2015

Academic Editor: Justin Dauwels

Copyright (c) 2015 Xusheng Ai et al. This is an open access article distributed under the Creative Commons Attribution License, which permits unrestricted use, distribution, and reproduction in any medium, provided the original work is properly cited.

\begin{abstract}
To improve the classification performance of imbalanced learning, a novel oversampling method, immune centroids oversampling technique (ICOTE) based on an immune network, is proposed. ICOTE generates a set of immune centroids to broaden the decision regions of the minority class space. The representative immune centroids are regarded as synthetic examples in order to resolve the imbalance problem. We utilize an artificial immune network to generate synthetic examples on clusters with high data densities, which can address the problem of synthetic minority oversampling technique (SMOTE), which lacks reflection on groups of training examples. Meanwhile, we further improve the performance of ICOTE via integrating ENN with ICOTE, that is, ICOTE + ENN. ENN disposes the majority class examples that invade the minority class space, so ICOTE + ENN favors the separation of both classes. Our comprehensive experimental results show that two proposed oversampling methods can achieve better performance than the renowned resampling methods.
\end{abstract}

\section{Introduction}

The class imbalance problem typically occurs when, in a binary classification problem, there are more training examples of one class than those of the other class. This situation is known as the class imbalance problem [1]. Under the circumstances, most standard algorithms fail to properly represent the distributive characteristics of complex imbalanced datasets and then provide unfavorable accuracies across examples of two classes [2]. Furthermore, it is worth pointing out that the minority class is usually the one that has the highest interest from a learning point of view and it also implies a great cost when it is not well classified [3].

Standard classification learning algorithms are often biased towards the majority class (known as the negative class). Therefore, it is not unusual that there is a higher misclassification rate for the minority class (i.e., the positive class) instances. In order to deal with this problem, a large number of approaches have been proposed to counter the sparsity in the distribution. Among them, the "synthetic minority oversampling technique" (SMOTE) [4] has become one of the most renowned approaches in this area. It can be essential to provide new related information on the positive class to the learning algorithm, in addition to undersampling majority class, which is completely different from undersampling majority class. Batista et al. proposed an integration method called SMOTE + ENN [5], which uses Wilson's edited nearest neighbor (denoted as ENN) rule [6] to remove examples whose classes differ from the classes of at least a half of their nearest neighbors. Han et al. present two new minority oversampling methods, borderline-SMOTE1 and borderlineSMOTE2 [7], in which only the minority class examples near the borderline are oversampled. Later, Bunkhumpornpat et al. published safe-level-SMOTE [8]. Their approach samples minority instances along the safe level that is computed using nearest neighboring minority instances. Ramentol et al. came up with another oversampling method with the application of an editing technique based on the rough set theory and the lower approximation of a subset [9].

In this paper we present an immune centroids oversampling technique (ICOTE) based on immune network theory. We utilize an aiNet model [10] to generate immune centroids 
of clusters of high data density. Our work resamples the minority class by introducing immune centroids of clusters of minority class examples. The resampling creates larger but less specific decision regions. Meanwhile, we also integrate our ICOTE with ENN together, that is, ICOTE + ENN. ICOTE + ENN can not only sample minority class examples but also dispose majority class examples that invade the minority class space. We expect that this hybrid method excels ICOTE in terms of the separation of both classes. Our experimental results show that both ICOTE and ICOTE + ENN achieve better performance in the application of three paradigms, comparing with the existing methods.

The rest of this paper is organized as follows. We review related work in Section 2. Section 3 presents our proposed oversampling methods ICOTE and ICOTE + ENN. Our experimental results and comparisons are shown in Section 4. Finally, we conclude this paper in Section 5.

\section{Related Work}

In order to deal with imbalanced issues, some articles studied different resampling techniques, which change the class distribution. These articles empirically showed that applying a preprocessing step in order to balance the class distribution usually is a useful solution [5, 11-13]. Furthermore, the main advantage of these techniques is that they are independent of the underlying classifier. Resampling techniques can be categorized into three groups or families:

(1) undersampling methods, which create a subset of the original dataset by eliminating some instances (usually majority class instances),

(2) oversampling methods, which create a superset of the original dataset by replicating some instances or creating new instances from the existing ones,

(3) hybrids methods, which combine both sampling approaches from the above.

López et al. [14] evaluated various sampling methodologies on a variety of datasets with different class distributions. They selected a collection of methods belonging to the three categories. They concluded that both SMOTE [4] and SMOTE + ENN [5] are more applicable and give very good results for datasets with various imbalanced rates. They also noted that the sophisticated sampling techniques did not give any clear advantage in the domains considered.

The SMOTE algorithm [4] oversamples the minority class. Specifically, it introduces synthetic examples along the line segments through joining some/all of the $k$ minority class nearest neighbors. Depending on the amount of oversampling required, neighbors from the $k$-nearest neighbors are randomly chosen. Figures 1-2 illustrate the distribution change in the application of SMOTE [4].

SMOTE + ENN [5] uses ENN to remove examples from both classes. Since some majority class examples might invade the minority class space and vice versa, SMOTE + ENN [5] reduces the possibility of overfitting introduced by synthetic examples. The cleaning result of ENN is illustrated in Figure 3.

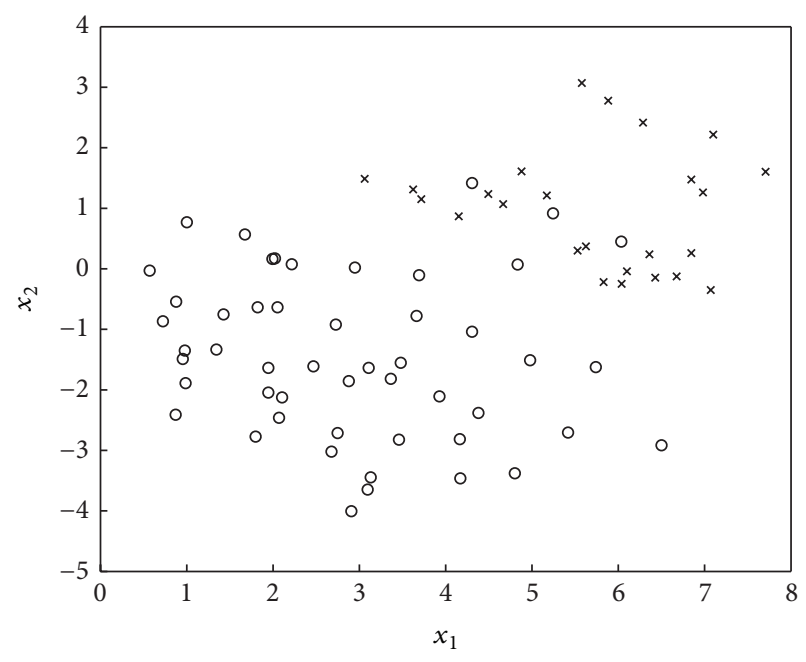

FIGURE 1: Data sample composed of 50 majority class examples and 25 minority class examples.

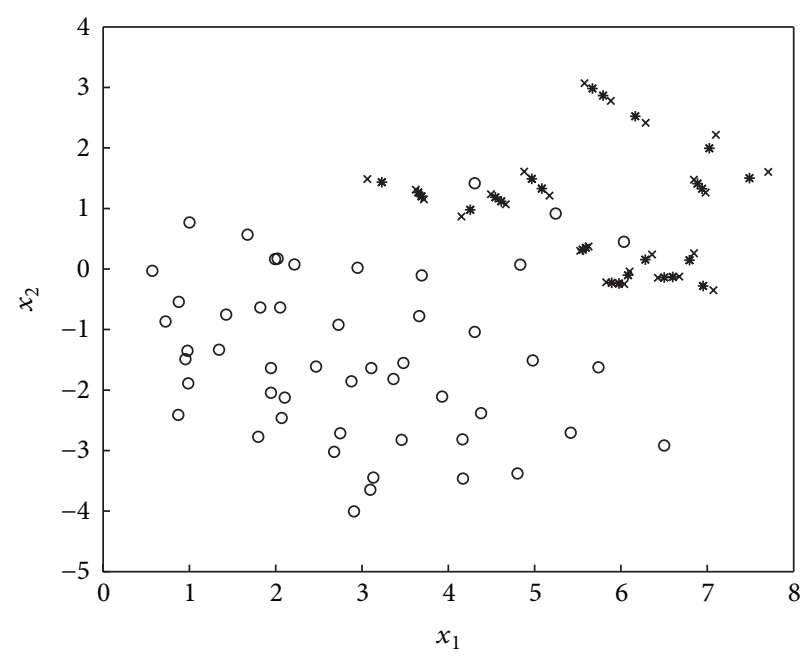

FIGURE 2: SMOTE introduces each synthetic example (in star) along the line segment joining the 2 minority class neighbors.

Jo and Japkowicz [15] discussed whether class imbalance is truly responsible for this degradation or whether it can be explained in some other ways. Their experiments suggest that the problem is not directly caused by class imbalance, but rather that class imbalances may yield small groups which, in turn, will cause this degradation. SMOTE [4] and its successors enrich the minority class space without considering data intrinsic characteristics such as small groups. The SMOTEbased methods might create the synthetic examples which underrepresent actual clusters or are attributed to noisy data. We will describe how our method overcomes the inherent drawback in the subsequent section.

\section{Our Methods}

In this section, we first briefly introduce the basic concepts and knowledge of immune systems. After that, we present 


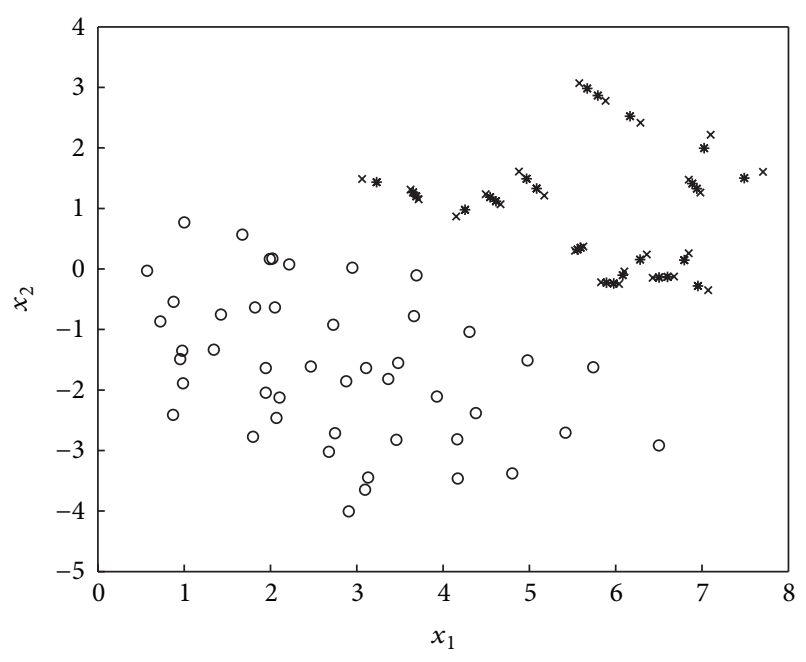

FIGURE 3: SMOTE introduces synthetic examples (in star) along the line segments via joining 2 minority class neighbors and ENN disposes the majority class examples in the minority class space.

our oversampling method ICOTE based on immune network theory and its improved version ICOTE + ENN.

3.1. Immune Systems. Before discussing our method, we sketch a few aspects of the human adaptive immune system. The immune systems guard our bodies against infections due to the attacks of antigens. The surface receptors on Bcells (one kind of lymphocyte) are able to recognize specific antigens. The response of a receptor to an antigen can activate its hosting B-cell. Activated B-cell then proliferates and differentiates into memory cells. Memory cells secrete antibodies to neutralize the pathogens through complementary pattern matching. During the proliferation of the activated B-cells, a mutation mechanism is employed to create diverse antibodies by altering the gene segments. Some of the mutants may be a better match for the corresponding antigen. In order to be protective, the immune system must learn to distinguish between our own (self) cells and malefic external (nonself) invaders. This process is called self/nonself discrimination: those cells recognized as self do not promote an immune response, and the system is said to be tolerant to them, while those that are not provoke a reaction resulting in their elimination.

The immune network theory, as originally proposed in [16], hypothesizes a novel viewpoint of lymphocyte activities, natural antibody production, preimmune repertoire selection, tolerance and self/nonself discrimination, memory, and the evolution of the immune system. It was suggested that the immune system is composed of a regulated network of cells and molecules that recognize one another. The immune cells can respond either positively or negatively to the recognition signal (antigen or another immune cell or molecule). A positive response would result in cell proliferation, cell activation, and antibody secretion, while a negative response would lead to tolerance and suppression.
Learning in the immune system involves raising the population size and affinity of those lymphocytes that have proven themselves valuable by having recognized any antigen. Burnet [17] introduced clonal selection theory by modifying Jerne's theory. The theory stated that, in a preexisting group of lymphocytes (specifically B-cells), a specific antigen only activates (i.e., selection) its counter-specific cell so that a particular cell is induced to multiply (producing its clones) for antibody production. With repeated exposures to the same antigen, the immune system produces antibodies of successively greater affinities. A secondary response elicits antibodies with greater affinity than in a primary response. Based on the clonal selection principle, de Castro and von Zuben [18] proposed a computational implementation of the clonal selection principle that explicitly takes into account the affinity maturation of the immune response. He also defined aiNet (an artificial immune network model) for data analysis [10]. The aiNet is an edge-weighted graph, not necessarily fully connected, composed of a set of nodes, called antibodies, and sets of node pairs called edges with an assigned number called weight, or connection strength, associated with each connected edge. The aiNet clusters serve as internal images (mirrors) responsible for mapping the existing clusters in the dataset into network clusters. These clusters map those of the original dataset. The shape of the spatial distribution of antibodies follows the shape of the antigenic spatial distribution.

3.2. Immune Centroids Resampling. In this paper we present a resampling method based on immune network theory. We use the aiNet model [10] to generate antibody-derived synthetic examples and extend a training set to balance sample distribution. The immune synthetic examples represent internal images of original minority class examples, so we call the resampling method immune centroids oversampling technique (ICOTE).

Before explaining ICOTE, we introduce some notations to describe the resampling method. Given a set of $n$ labeled examples $D_{n}=\left\{\left(\overrightarrow{X_{1}}, Y_{1}\right), \ldots,\left(\overrightarrow{X_{n}}, Y_{n}\right)\right\}$ with input vectors $\vec{X}_{i} \in R^{d}$ and class label $Y_{i} \in\{$ negative, positive $\}$, we measure the affinity (complementarity level) of the antigen-antibody match using Euclidean distance. As we know, the Euclidean distance of two vectors is

$$
\operatorname{dist}\left(\vec{X}_{i}, \vec{X}_{j}\right)=\sqrt{\sum\left(X_{i}^{m}-X_{j}^{m}\right)^{2}}
$$

where $m$ is the dimension of each vector. The antigenantibody affinity is inversely proportional to the Euclidean distance. The smaller the distance, the higher the affinity, and vice versa.

Our ICOTE includes four major steps as follows. 
3.2.1. Attribute Selection. In order to reduce computational cost, we first remove the attributes whose values are constant:

$$
\begin{array}{r}
\text { fselect }(\vec{X})=\left(X^{1}, \ldots, X^{k}, X^{k+l+1}, \ldots, X^{d}\right)^{T}, \\
X^{k+1} \equiv C_{1}, \ldots, X^{k+l}=C_{l}, \quad C_{i} \in R .
\end{array}
$$

3.2.2. Unit-Based Normalization. Then we adjust the values of attributes on different scales to a notionally common scale $[0,1]$ :

$$
\operatorname{norm}(x)=\frac{x-x_{\min }}{x_{\max }-x_{\min }}
$$

3.2.3. Immune Centroids Generation. There are three steps for generating immune centroids. First, the selected antibodies $\overrightarrow{A b}$ are going to proliferate (clone) proportionally to their antigenic affinity: the higher the affinity, the larger the clone size $n c$ for each selected antibody:

$$
\text { clone }(\overrightarrow{A b})=\left\{\overrightarrow{A b_{1}}, \ldots, \overrightarrow{A b_{n c}}\right\}
$$

Next, each antibody $\overrightarrow{A b}$ from the clone set will suffer a mutation with a rate $\alpha_{k}$, which is inversely proportional to the antigenic affinity of its parent antibody $\overrightarrow{\mathrm{Ag}}$ :

$$
\begin{aligned}
\operatorname{mutate}(\overrightarrow{A b}) & =\overrightarrow{A b}+\alpha_{k}(\overrightarrow{A b}-\overrightarrow{A g}), \\
\alpha_{k} & =\frac{1}{d} * \operatorname{dist}(\overrightarrow{A b}, \overrightarrow{A g}) .
\end{aligned}
$$

And then we eliminate the memory antibodies (denoted as $M$ ) with a low antigen-antibody affinity (clonal suppression) $f_{i j}$ and a high antibody-antibody affinity (network suppression) $f_{i j}^{\prime}$ :

$$
\operatorname{suppress}(M)=M-M_{f_{i j}>T_{1}}-M_{f_{i j}^{\prime}>T_{2}}, \quad T_{1} \in R, T_{2} \in R \text {. }
$$

3.2.4. Denormalization. Next, we denormalize memory antibodies $M$ and make synthetic examples identical to sample distribution:

$$
\operatorname{de}-\operatorname{norm}(x)=x_{\min }+\left(x_{\max }-x_{\min }\right) x
$$

3.2.5. Attribute Replacement. At the end, we put back constant-value attributes:

$$
\begin{array}{r}
\operatorname{de}-f \operatorname{select}(\vec{S})=\left(S^{1}, \ldots, S^{k}, C_{1}, \ldots, C_{l}, X^{k+l+1}, \ldots, X^{d}\right)^{T}, \\
C_{i} \in R .
\end{array}
$$

Correspondingly, the algorithm is described as shown in Algorithm 1.

ICOTE samples minority class examples to generate memory antibodies (immune centroids). The shape of

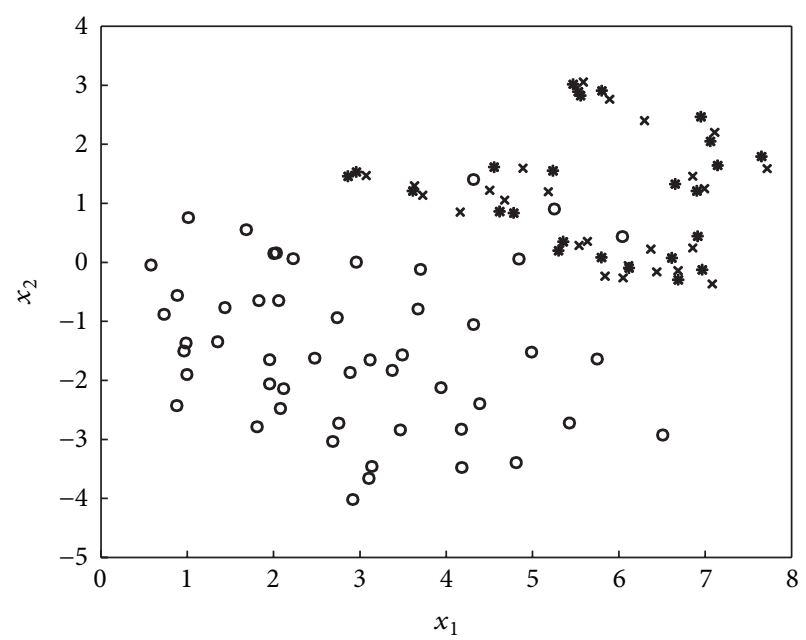

Figure 4: ICOTE introduces the immune centroids (in star) following the shape of the neighboring minority class examples.

the spatial distribution of the immune centroids follows that of the minority class examples. Therefore, it avoids more small groups or outliers introduced by oversampling. For instance, we depict the immune centroids in Figure 4. Intuitively, each of them in star shares the same group with one or several neighboring minority class examples. Introducing immune centroids for learning not only creates larger and less specific decision regions but also decreases the likelihood of overfitting occurring, which is the major drawback of SMOTE [4].

We also propose an integrated method called ICOTE + ENN, integrating ICOTE with the Wilson's edited nearest neighbor rule (i.e., ENN) [6]. In this integrated method, ICOTE oversamples minority class examples, and ENN discards "dirty" examples deviating from the majority class space. When the class of an example differs from the class of more than a half of the nearest neighbors, the example will be removed from the training set. The result of the integrated method is illustrated in Figure 5. Figure 5 shows that the hybrid method makes the two class spaces separated. In the next section, we will show our empirical results for our two methods.

\section{Experiments}

In this section, we will investigate the performance of our proposed oversampling methods ICOTE and ICOTE + ICOTE and compare them with the existing well-known oversampling methods.

4.1. Experimental Settings. Our experiments are conducted based on three base classifiers: $k \mathrm{NN}, \mathrm{C} 4.5$, and SVM. We use these algorithms, since they are available within the KEEL software tool [19]. In the experiments, the parameter values are set based on the recommendations from the corresponding authors. The specific settings are as follows. 
Input:

Sample[][] array for original minority examples, $R$ number of original minority examples, $K$ initial antibody number, $N$ maximum number of generations, $M[][]$ array for memory antibodies, $A b[][]$ array for network antibodies,

$\mathrm{Ag}[][]$ array for antigens, Dist[] array for Euclidian distances

Output: Synthetic[][] Array for synthetic minority examples

for $i=1$ to $R$

end for

Compute: Sample $[i]=$ fselect $($ Sample$[i]) / /$ Use formula (2)

for $i=1$ to $R$

end for

Compute: $A g[i]=\operatorname{norm}($ Sample $[i]) / /$ Use formula $(3)$

Generate $K$ random antibodies $A b[][]$

while $N<10$ do

Initialize memory antibodies $M[][]$

for $i=1$ to $R$

for $j=1$ to $K$

Compute: $\operatorname{Dist}[]=\operatorname{dist}(A g[i], A b[j]) / /$ Use formula (1)

end for

Clone $K$ antibodies in proportion to antigen-antibody affinities // Use formula (4)

Select a portion of antibodies to perform mutation // Use formula (5)

Dispose antibodies with antigen-antibody affinity $<0.05 / /$ Use formula (6)

Append antibodies to $M[][]$

end for

Compute: mnum = number of memory antibodies $M[][]$

for $i=1$ to mnum

Compute: $\operatorname{Dist}[]=\operatorname{dist}(M[i], M[i]) / /$ Use formula (1)

end for

Dispose memory antibodies with antibody-antibody affinity $<0.05$ // Use formula (6)

Fill $A b[][]$ with $M[][]$ and new $K$ random antibodies

$N=N+1$

end for

Compute: mnum $=$ number of memory antibodies $M[][]$

for $i=1$ to mnum

Compute: Synthetic $[i]=\operatorname{de}-\operatorname{norm}(M[i]) / /$ Use formula $(7)$

end for

for $i=1$ to mnum

Compute: Synthetic $[i]=\operatorname{de}-$ fselect $($ Synthetic $[i]) / /$ Use formula (8)

end for

Algorithm 1

(1) Instance based learning $(k N N)$ [20]: in this algorithm, we set $k=1$ and use the Euclidean distance metric.

(2) C4.5 decision tree [21]: for C4.5, we set a confidence level as 0.25 and the minimum number of item sets per leaf as 2 and use pruning.

(3) Support vector machines (SVM) [22]: for SVM, we choose Polykernel reference functions, setting an internal parameter 1.0 for the exponent of each kernel function and a penalty parameter of the error term as 1.0 .

We conduct experiments on 38 datasets from the KEEL dataset repository [23], whose characteristics are summarized in Table 1, namely, the number of examples (\#Ex.), number of attributes (\#Atts.), and instance ratio (IR). The experiments are evaluated in terms of one of the popular metrics, the area under the ROC curve (AUC) [24, 25]. The experimental results are obtained based on 5-fold cross-validation.
We choose 5-fold cross-validation, because it can keep sufficient positive class instances in different folds. Thus, we can avoid additional problems in the data distribution which were discussed in $[26,27]$, especially for highly imbalanced datasets.

We must point out that the dataset partitions employed in this paper are available from the KEEL dataset repository [23], so that researchers can use the same data partitions for comparisons.

4.2. Evaluation in Imbalanced Domains. In imbalanced domains, a well-known approach to unify these measures and to produce an evaluation criterion is to use the receiver operating characteristic (ROC) graphic [24]. This graphic allows the visualization of the trade-off between the benefits $\left(\mathrm{TP}_{\text {rate }}\right)$ and costs $\left(\mathrm{FP}_{\text {rate }}\right)$, as it evidences that any classifier cannot increase the number of true positives without also increasing the false positives. The area under the ROC curve (AUC) [25] corresponds to the probability of correctly 
TABLE 1: The characteristics of imbalanced datasets.

\begin{tabular}{|c|c|c|c|c|c|c|c|}
\hline Name & \#Ex. & \#Attrs & IR & Name & \#Ex. & \#Attrs & IR \\
\hline glass1 & 214 & 9 & 1.82 & yeast-2_vs_4 & 514 & 8 & 9.08 \\
\hline ecoli-0_vs_1 & 220 & 7 & 1.86 & yeast-0-5-6-7-9_vs_4 & 528 & 8 & 9.35 \\
\hline wisconsinimb & 683 & 9 & 1.86 & vowel0 & 988 & 13 & 10.1 \\
\hline pimaimb & 768 & 8 & 1.9 & glass-0-1-6_vs_2 & 192 & 9 & 10.29 \\
\hline iris0 & 150 & 4 & 2 & glass2 & 214 & 9 & 10.39 \\
\hline glass0 & 214 & 9 & 2.06 & shuttle-c0-vs-c4 & 1829 & 9 & 13.87 \\
\hline yeast1 & 1484 & 8 & 2.46 & yeast-1_vs_7 & 459 & 8 & 13.87 \\
\hline habermanimb & 306 & 3 & 2.68 & glass4 & 214 & 9 & 15.47 \\
\hline vehicle2 & 846 & 18 & 2.52 & ecoli4 & 336 & 7 & 13.84 \\
\hline vehiclel & 846 & 18 & 2.52 & glass-0-1-6_vs_5 & 184 & 9 & 19.44 \\
\hline vehicle3 & 846 & 18 & 2.52 & shuttle-c2-vs-c4 & 129 & 9 & 20.5 \\
\hline glass-0-1-2-3_vs_4-5-6 & 214 & 9 & 3.19 & yeast-1-4-5-8_vs_7 & 693 & 8 & 22.1 \\
\hline vehicle 0 & 846 & 18 & 3.23 & glass5 & 214 & 9 & 22.81 \\
\hline ecoli1 & 336 & 7 & 3.36 & yeast-2_vs_8 & 482 & 8 & 23.1 \\
\hline newthyroid2 & 215 & 5 & 4.92 & yeast 4 & 1484 & 8 & 28.41 \\
\hline new-thyroid1 & 215 & 5 & 5.14 & yeast-1-2-8-9_vs_7 & 947 & 8 & 30.56 \\
\hline ecoli2 & 336 & 7 & 5.46 & yeast5 & 1484 & 8 & 32.78 \\
\hline glass6 & 214 & 9 & 6.38 & ecoli-0-1-3-7_vs_2-6 & 281 & 7 & 39.15 \\
\hline yeast3 & 1484 & 8 & 8.11 & yeast6 & 1484 & 8 & 39.15 \\
\hline
\end{tabular}

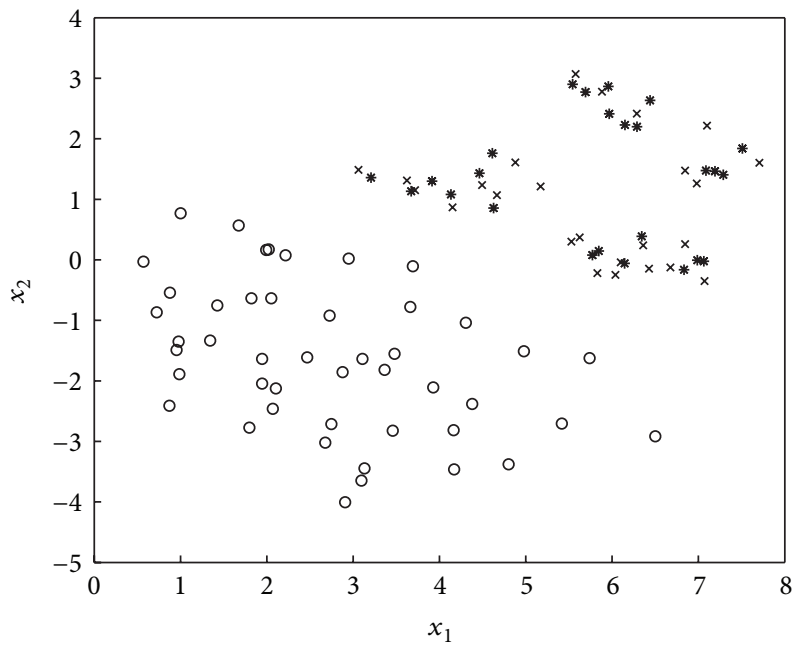

Figure 5: ICOTE introduces the immune centroids (in star) following the shape of the neighboring minority class examples and ENN [6] disposes the majority class examples in the minority class space.

identifying which one of the two stimuli is noise and which one is signal plus noise. The AUC provides a single measure of a classifier's performance for evaluating which model is better on average. The AUC measure is computed just by obtaining the area of the graphic:

$$
\mathrm{AUC}=\frac{1+\mathrm{TP}_{\text {rate }}-\mathrm{FP}_{\text {rate }}}{2} .
$$

TABLE 2: Average AUC results of different resampling methods with $k \mathrm{NN}, \mathrm{C} 4.5$, and SVM as the base learner, respectively.

\begin{tabular}{lccc}
\hline & $k N N$ & $C 45$ & $S V M$ \\
\hline None & 0.791 & 0.793 & 0.712 \\
SMOTE & 0.839 & 0.839 & 0.850 \\
SMOTE + ENN & 0.848 & 0.840 & 0.850 \\
SL-SMOTE & 0.836 & 0.828 & 0.844 \\
ADASYN & 0.804 & 0.819 & 0.843 \\
ICOTE & $\mathbf{0 . 9 5 5}$ & $\mathbf{0 . 9 3 6}$ & $\mathbf{0 . 8 7 1}$ \\
ICOTE + ENN & $\mathbf{0 . 9 6 9}$ & $\mathbf{0 . 9 4 7}$ & $\mathbf{0 . 8 8 7}$ \\
\hline
\end{tabular}

AUC combines the individual measures of both the positive and negative classes so that we can utilize it to measure quality results of different paradigms for imbalanced data.

4.3. Experimental Results. In this section, we investigate the performance of the resampling methods on the imbalanced datasets listed in Table 1.

As shown in the previous work [14, Table 4] on the keel datasets, SMOTE [4] and SMOTE + ENN [5] have the highest rank for the three classification algorithms $(k \mathrm{NN}$, C4.5, and SVM) used in their study, and both ADASYS [28] and SL-SMOTE [29] achieve the 2nd highest AUC values. So we select these four resampling algorithms and compare our ICOTE and ICOTE + ENN with them. The average AUC results of different resampling methods with three base learners $k \mathrm{NN}, \mathrm{C} 4.5$, and SVM over all 38 datasets are shown in Table 2. Besides, we also have the experimental results obtained based on the three base learners directly without using resampling techniques, which is denoted as "none" in 
TABLE 3: The Shaffer test for the oversampling techniques with $k N N$ using the AUC measure.

\begin{tabular}{lccccccc}
\hline & None & SMOTE & SMOTE + ENN & SL-SMOTE & ADASYN & ICOTE & ICOTE + ENN \\
\hline None & $\mathrm{X}$ & $-(0.001)$ & $=(0.000)$ & $=(0.009)$ & $=(0.957)$ & $-(0.000)$ & $-(0.000)$ \\
SMOTE & $+(0.001)$ & $\mathrm{X}$ & $=(0.633)$ & $=(0.441)$ & $+(0.001)$ & $-(0.000)$ & $-(0.000)$ \\
SMOTE + ENN & $=(0.000)$ & $=(0.633)$ & $\mathrm{X}$ & $=(0.212)$ & $+(0.000)$ & $-(0.000)$ & $-(0.000)$ \\
SL-SMOTE & $=(0.009)$ & $=(0.441)$ & $=(0.212)$ & $\mathrm{X}$ & $=(0.010)$ & $-(0.000)$ & $-(0.000)$ \\
ADASYN & $=(0.957)$ & $-(0.001)$ & $-(0.000)$ & $=(0.010)$ & $\mathrm{X}$ & $-(0.000)$ & $-(0.000)$ \\
ICOTE & $+(0.000)$ & $+(0.000)$ & $+(0.000)$ & $+(0.000)$ & $+(0.000)$ & $\mathrm{X}$ & $=(0.167)$ \\
ICOTE + ENN & $+(0.000)$ & $+(0.000)$ & $+(0.000)$ & $+(0.000)$ & $+(0.000)$ & $=(0.167)$ & $\mathrm{X}$ \\
\hline
\end{tabular}

TABLE 4: The Shaffer test for the oversampling techniques with C4.5 using the AUC measure.

\begin{tabular}{lccccccc}
\hline & None & SMOTE & SMOTE + ENN & SL-SMOTE & ADASYN & ICOTE & ICOTE + ENN \\
\hline None & $\mathrm{X}$ & $-(0.00)$ & $-(0.000)$ & $=(0.117)$ & $=(0.193)$ & $-(0.000)$ & $-(0.000)$ \\
SMOTE & $+(0.001)$ & $\mathrm{X}$ & $=(0.541)$ & $=(0.300)$ & $=(0.193)$ & $-(0.000)$ & $-(0.000)$ \\
SMOTE + ENN & $+(0.000)$ & $=(0.560)$ & $\mathrm{X}$ & $=(0.009)$ & $=(0.056)$ & $-(0.000)$ & $-(0.000)$ \\
SL-SMOTE & $=(0.117)$ & $=(0.300)$ & $=(0.009)$ & $\mathrm{X}$ & $=(0.790)$ & $-(0.000)$ & $-(0.000)$ \\
ADASYN & $=(0.193)$ & $=(0.193)$ & $=(0.056)$ & $=(0.790)$ & $\mathrm{X}$ & $-(0.000)$ & $-(0.000)$ \\
ICOTE & $+(0.000)$ & $+(0.000)$ & $+(0.000)$ & $+(0.000)$ & $+(0.000)$ & $\mathrm{X}$ & $=(0.353)$ \\
ICOTE + ENN & $+(0.000)$ & $+(0.000)$ & $+(0.000)$ & $+(0.000)$ & $+(0.000)$ & $=(0.353)$ & $\mathrm{X}$ \\
\hline
\end{tabular}

Table 2. Please note that our experimental results on each dataset are shown in the Appendix.

From Table 2, we can see that our methods ICOTE and ICOTE + ENN perform much better than the other four resampling methods, on all three base learners. And ICOTE + ENN does improve the performance of ICOTE. Our experimental results also show that SMOTE and SMOTE + ENN perform better than SL-SMOTE and ADASYN. SMOTE + ENN does improve the performance of SMOTE a little on all the three base learners. Between SL-SMOTE and ADASYN, SL-SMOTE performs better. That is, ADASYN is the worst among the six resampling methods.

Besides the average results shown in Table 2, we also rank the resampling methods on each dataset with each base learner. The average ranks of each method with each base learner are shown in Figure 6. From Figure 6, we can see that the average rank of ICOTE + ENN is the best under any one of the three base learners. ICOTE ranks the second consistently. SMOTE + ENN always ranks the third. SMOTE always ranks the fourth. It is obvious that "none" (without using resampling techniques) performs the worst when either C4.5 or SVM is used as the base learner. Between SLSMOTE and ADASYN, SL-SMOTE always performs better than ADASYN. These conclusions are consistent with the conclusions we made based on the average AUC, shown in Table 2.

For the sake of finding out which algorithms are distinctive among the pair comparisons of these methods, we carry out a Shaffer post hoc test [30], which is shown in Tables 3-5. In these tables, a "+" symbol implies that the algorithm in the row is statistically better than the one in the column, "-" implies the contrary, and "=" means that the two algorithms

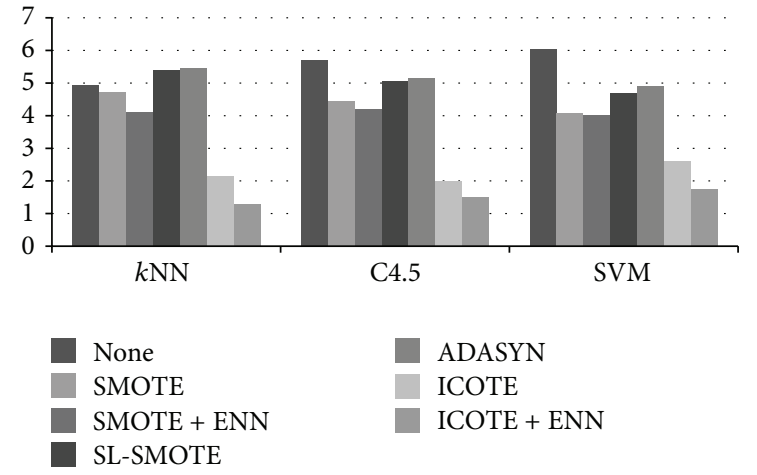

FIGURE 6: The average ranks of the resampling methods under three base learners, respectively.

compared show no significant difference. In brackets, the unadjusted $P$ value associated with each comparison is also presented. Shaffer's procedure rejects those hypotheses that have an unadjusted $P$ value $\leq 0.002$.

In order to explain why ICOTE and ICOTE + ENN obtain the highest performance, we may emphasize two feasible reasons. The first one is related to the addition of significant information within the minority class examples by including immune centroids of clusters. These immune centroids allow the formation of larger clusters to help the classifiers to separate both classes, and its cleaning procedure also adds benefits to the generalization ability during learning. The second reason is that the immune centroids represent inherent clusters of the minority class examples and overcome 
TABLE 5: The Shaffer test for the oversampling techniques with SVM using the AUC measure.

\begin{tabular}{lccccccc}
\hline & None & SMOTE & SMOTE + ENN & SL-SMOTE & ADASYN & ICOTE & ICOTE + ENN \\
\hline None & $\mathrm{X}$ & $-(0.000)$ & $-(0.000)$ & $=(0.005)$ & $=(0.009)$ & $-(0.000)$ & $-(0.000)$ \\
SMOTE & $+(0.000)$ & $\mathrm{X}$ & $=(0.710)$ & $=(0.352)$ & $=(0.254)$ & $-(0.001)$ & $-(0.000)$ \\
SMOTE + ENN & $+(0.000)$ & $=(0.710)$ & $\mathrm{X}$ & $=(0.193)$ & $=(0.130)$ & $=(0.005)$ & $-(0.000)$ \\
SL-SMOTE & $=(0.005)$ & $=(0.352)$ & $=(0.193)$ & $\mathrm{X}$ & $=(0.831)$ & $=(0.005)$ & $-(0.000)$ \\
ADASYN & $=(0.009)$ & $=(0.254)$ & $=(0.130)$ & $=(0.831)$ & $\mathrm{X}$ & $-(0.000)$ & $-(0.000)$ \\
ICOTE & $+(0.000)$ & $+(0.001)$ & $=(0.005)$ & $+(0.000)$ & $+(0.000)$ & $\mathrm{X}$ & $=(0.080)$ \\
ICOTE + ENN & $+(0.000)$ & $+(0.000)$ & $+(0.000)$ & $+(0.000)$ & $+(0.000)$ & $=(0.080)$ & $\mathrm{X}$ \\
\hline
\end{tabular}

TABLE 6: AUC results of different resampling methods with $k \mathrm{NN}$ as the base learner.

\begin{tabular}{|c|c|c|c|c|c|c|c|}
\hline & None & SMOTE & SMOTE + ENN & SL-SMOTE & ADASYN & ICOTE & ICOTE + ENN \\
\hline glass1 & 0.789 & 0.781 & 0.776 & 0.778 & 0.789 & 0.869 & 0.858 \\
\hline ecoli-0_vs_1 & 0.963 & 0.970 & 0.973 & 0.969 & 0.963 & 0.970 & 0.971 \\
\hline wisconsinimb & 0.955 & 0.965 & 0.975 & 0.964 & 0.957 & 0.982 & 0.979 \\
\hline pimaimb & 0.664 & 0.664 & 0.703 & 0.692 & 0.664 & 0.796 & 0.832 \\
\hline iris0 & 1.000 & 1.000 & 1.000 & 1.000 & 1.000 & 1.000 & 1.000 \\
\hline glass 0 & 0.827 & 0.835 & 0.832 & 0.831 & 0.827 & 0.913 & 0.926 \\
\hline yeast1 & 0.634 & 0.649 & 0.671 & 0.644 & 0.634 & 0.849 & 0.889 \\
\hline habermanimb & 0.547 & 0.582 & 0.612 & 0.565 & 0.547 & 0.764 & 0.807 \\
\hline vehicle2 & 0.940 & 0.938 & 0.936 & 0.938 & 0.938 & 0.975 & 0.983 \\
\hline vehiclel & 0.660 & 0.658 & 0.709 & 0.651 & 0.630 & 0.884 & 0.923 \\
\hline vehicle3 & 0.665 & 0.677 & 0.700 & 0.690 & 0.676 & 0.878 & 0.870 \\
\hline glass-0-1-2-3_vs_4-5-6 & 0.915 & 0.949 & 0.959 & 0.942 & 0.912 & 0.981 & 0.994 \\
\hline vehicle 0 & 0.911 & 0.931 & 0.920 & 0.925 & 0.910 & 0.982 & 0.986 \\
\hline ecoli1 & 0.859 & 0.865 & 0.881 & 0.864 & 0.797 & 0.942 & 0.991 \\
\hline new-thyroid2 & 0.949 & 0.967 & 0.961 & 0.981 & 0.980 & 0.992 & 0.997 \\
\hline new-thyroid1 & 0.949 & 0.955 & 0.946 & 0.983 & 0.977 & 0.992 & 0.994 \\
\hline ecoli2 & 0.862 & 0.931 & 0.926 & 0.909 & 0.906 & 0.972 & 0.996 \\
\hline glass6 & 0.813 & 0.888 & 0.874 & 0.888 & 0.871 & 0.992 & 0.992 \\
\hline yeast3 & 0.857 & 0.868 & 0.866 & 0.866 & 0.818 & 0.973 & 0.994 \\
\hline yeast-2_vs_4 & 0.833 & 0.902 & 0.877 & 0.875 & 0.852 & 0.981 & 1.000 \\
\hline yeast-0-5-6-7-9_vs_4 & 0.680 & 0.784 & 0.788 & 0.757 & 0.702 & 0.965 & 0.988 \\
\hline vowel0 & 0.971 & 0.998 & 0.999 & 0.996 & 1.000 & 1.000 & 1.000 \\
\hline glass-0-1-6_vs_2 & 0.619 & 0.665 & 0.647 & 0.684 & 0.577 & 0.954 & 0.976 \\
\hline glass2 & 0.669 & 0.690 & 0.738 & 0.696 & 0.601 & 0.962 & 0.977 \\
\hline shuttle-c0-vs-c4 & 1.000 & 0.996 & 0.996 & 0.996 & 0.996 & 1.000 & 1.000 \\
\hline yeast-1_vs_7 & 0.594 & 0.713 & 0.727 & 0.738 & 0.646 & 0.972 & 0.982 \\
\hline glass4 & 0.793 & 0.894 & 0.915 & 0.882 & 0.818 & 0.980 & 0.995 \\
\hline ecoli4 & 0.814 & 0.961 & 0.928 & 0.881 & 0.870 & 0.992 & 0.997 \\
\hline glass-0-1-6_vs_5 & 0.891 & 0.874 & 0.921 & 0.871 & 0.836 & 0.989 & 0.997 \\
\hline shuttle-c2-vs-c4 & 1.000 & 0.996 & 0.996 & 1.000 & 0.950 & 1.000 & 1.000 \\
\hline yeast-1-4-5-8_vs_7 & 0.500 & 0.628 & 0.675 & 0.642 & 0.574 & 0.972 & 0.986 \\
\hline glass5 & 0.898 & 0.921 & 0.921 & 0.918 & 0.893 & 0.990 & 0.995 \\
\hline yeast-2_vs_8 & 0.500 & 0.798 & 0.816 & 0.781 & 0.768 & 0.985 & 0.994 \\
\hline yeast 4 & 0.595 & 0.766 & 0.797 & 0.720 & 0.667 & 0.918 & 0.957 \\
\hline yeast-1-2-8-9_vs_7 & 0.616 & 0.601 & 0.622 & 0.595 & 0.553 & 0.975 & 0.992 \\
\hline yeast 5 & 0.883 & 0.952 & 0.938 & 0.942 & 0.846 & 0.992 & 0.999 \\
\hline ecoli-0-1-3-7_vs_2-6 & 0.748 & 0.837 & 0.832 & 0.832 & 0.843 & 0.987 & 0.996 \\
\hline yeast6 & 0.712 & 0.865 & 0.856 & 0.867 & 0.748 & 0.987 & 0.996 \\
\hline
\end{tabular}


TABLE 7: AUC results of different resampling methods with C4.5 as the base learner.

\begin{tabular}{|c|c|c|c|c|c|c|c|}
\hline & None & SMOTE & SMOTE + ENN & SL-SMOTE & ADASYN & ICOTE & $\mathrm{ICOTE}+\mathrm{ENN}$ \\
\hline glass1 & 0.733 & 0.695 & 0.718 & 0.726 & 0.727 & 0.790 & 0.783 \\
\hline ecoli-0_vs_1 & 0.983 & 0.983 & 0.983 & 0.983 & 0.959 & 0.975 & 0.949 \\
\hline wisconsinimb & 0.948 & 0.960 & 0.940 & 0.943 & 0.952 & 0.967 & 0.968 \\
\hline pimaimb & 0.703 & 0.725 & 0.712 & 0.714 & 0.705 & 0.763 & 0.837 \\
\hline iris0 & 0.990 & 0.990 & 0.990 & 0.990 & 0.990 & 0.990 & 0.995 \\
\hline glass0 & 0.817 & 0.782 & 0.808 & 0.756 & 0.788 & 0.851 & 0.871 \\
\hline yeast1 & 0.668 & 0.710 & 0.710 & 0.719 & 0.696 & 0.834 & 0.829 \\
\hline habermanimb & 0.576 & 0.636 & 0.649 & 0.632 & 0.637 & 0.758 & 0.807 \\
\hline vehicle2 & 0.949 & 0.959 & 0.952 & 0.945 & 0.939 & 0.966 & 0.962 \\
\hline vehiclel & 0.660 & 0.695 & 0.754 & 0.733 & 0.698 & 0.810 & 0.869 \\
\hline vehicle3 & 0.665 & 0.708 & 0.716 & 0.743 & 0.731 & 0.845 & 0.829 \\
\hline glass-0-1-2-3_vs_4-5-6 & 0.915 & 0.886 & 0.916 & 0.949 & 0.884 & 0.957 & 0.965 \\
\hline vehicle0 & 0.925 & 0.936 & 0.943 & 0.927 & 0.939 & 0.957 & 0.973 \\
\hline ecoli1 & 0.859 & 0.893 & 0.872 & 0.901 & 0.888 & 0.917 & 0.951 \\
\hline new-thyroid2 & 0.949 & 0.927 & 0.919 & 0.966 & 0.972 & 0.983 & 0.986 \\
\hline new-thyroidl & 0.949 & 0.938 & 0.952 & 0.930 & 0.955 & 0.981 & 0.977 \\
\hline ecoli2 & 0.862 & 0.894 & 0.877 & 0.872 & 0.902 & 0.940 & 0.942 \\
\hline glass6 & 0.813 & 0.892 & 0.917 & 0.863 & 0.853 & 0.954 & 0.975 \\
\hline yeast3 & 0.857 & 0.904 & 0.908 & 0.906 & 0.911 & 0.970 & 0.983 \\
\hline yeast-2_vs_4 & 0.833 & 0.895 & 0.883 & 0.859 & 0.865 & 0.974 & 0.980 \\
\hline yeast-0-5-6-7-9_vs_4 & 0.680 & 0.830 & 0.745 & 0.800 & 0.725 & 0.932 & 0.949 \\
\hline vowel0 & 0.971 & 0.979 & 0.988 & 0.959 & 0.969 & 0.992 & 0.990 \\
\hline glass-0-1-6_vs_2 & 0.619 & 0.712 & 0.625 & 0.741 & 0.580 & 0.911 & 0.969 \\
\hline glass2 & 0.669 & 0.687 & 0.756 & 0.753 & 0.730 & 0.929 & 0.954 \\
\hline shuttle-c0-vs-c4 & 1.000 & 0.999 & 1.000 & 0.994 & 0.999 & 0.999 & 1.000 \\
\hline yeast-1_vs_7 & 0.594 & 0.703 & 0.657 & 0.704 & 0.675 & 0.930 & 0.938 \\
\hline glass 4 & 0.793 & 0.855 & 0.915 & 0.816 & 0.824 & 0.965 & 0.979 \\
\hline ecoli4 & 0.814 & 0.951 & 0.923 & 0.882 & 0.876 & 0.987 & 0.987 \\
\hline glass-0-1-6_vs_5 & 0.891 & 0.957 & 0.919 & 0.796 & 0.930 & 0.994 & 0.997 \\
\hline shuttle-c2-vs-c4 & 1.000 & 0.996 & 1.000 & 0.922 & 0.996 & 1.000 & 1.000 \\
\hline yeast-1-4-5-8_vs_7 & 0.500 & 0.603 & 0.587 & 0.593 & 0.519 & 0.931 & 0.945 \\
\hline glass5 & 0.898 & 0.978 & 0.928 & 0.846 & 0.880 & 0.998 & 1.000 \\
\hline yeast-2_vs_8 & 0.500 & 0.824 & 0.844 & 0.814 & 0.653 & 0.987 & 0.985 \\
\hline yeast 4 & 0.595 & 0.750 & 0.783 & 0.780 & 0.734 & 0.922 & 0.923 \\
\hline yeast-1-2-8-9_vs_7 & 0.616 & 0.674 & 0.612 & 0.517 & 0.686 & 0.963 & 0.959 \\
\hline yeast5 & 0.883 & 0.941 & 0.972 & 0.947 & 0.927 & 0.991 & 0.995 \\
\hline ecoli-0-1-3-7_vs_2-6 & 0.748 & 0.619 & 0.717 & 0.717 & 0.623 & 0.985 & 0.989 \\
\hline yeast6 & 0.712 & 0.831 & 0.845 & 0.811 & 0.806 & 0.985 & 0.984 \\
\hline
\end{tabular}

the limitation that synthetic examples form new clusters or outliers.

\section{Conclusions}

In this paper we present two overresampling methods based on immune network theory. We draw the conclusions based on our experimental results and analyses as follows.

(1) ICOTE samples minority class examples to generate immune centroids as synthetic examples, which is far different from renowned resampling methods without considering sample architecture.
(2) ICOTE introduces minority class examples and ENN disposes majority class examples in the minority data space. ICOTE + ENN favors separating both classes.

(3) We compare our proposed methods ICOTE and ICOTE + ENN with representative resampling methods. Our experimental results showed that our approaches make significant improvement.

\section{Appendix}

See Tables 6, 7, and 8 . 
TABLE 8: AUC results of different resampling methods with SVM as the base learner.

\begin{tabular}{|c|c|c|c|c|c|c|c|}
\hline & None & SMOTE & SMOTE + ENN & SL-SMOTE & ADASYN & ICOTE & ICOTE + ENN \\
\hline glass1 & 0.496 & 0.612 & 0.651 & 0.612 & 0.609 & 0.630 & 0.570 \\
\hline ecoli-0_vs_1 & 0.967 & 0.980 & 0.983 & 0.980 & 0.962 & 0.982 & 0.949 \\
\hline wisconsinimb & 0.967 & 0.971 & 0.970 & 0.971 & 0.976 & 0.970 & 0.973 \\
\hline pimaimb & 0.719 & 0.742 & 0.742 & 0.750 & 0.747 & 0.745 & 0.821 \\
\hline iris0 & 1.000 & 1.000 & 1.000 & 1.000 & 1.000 & 1.000 & 1.000 \\
\hline glass0 & 0.691 & 0.752 & 0.749 & 0.734 & 0.735 & 0.760 & 0.806 \\
\hline yeast1 & 0.573 & 0.706 & 0.712 & 0.702 & 0.707 & 0.721 & 0.764 \\
\hline habermanimb & 0.504 & 0.626 & 0.645 & 0.622 & 0.626 & 0.636 & 0.756 \\
\hline vehicle2 & 0.953 & 0.950 & 0.953 & 0.944 & 0.950 & 0.960 & 0.962 \\
\hline vehiclel & 0.720 & 0.820 & 0.816 & 0.796 & 0.814 & 0.817 & 0.887 \\
\hline vehicle3 & 0.713 & 0.792 & 0.803 & 0.789 & 0.780 & 0.790 & 0.814 \\
\hline glass-0-1-2-3_vs_4-5-6 & 0.904 & 0.918 & 0.916 & 0.915 & 0.906 & 0.923 & 0.938 \\
\hline vehicle0 & 0.949 & 0.959 & 0.962 & 0.946 & 0.959 & 0.968 & 0.980 \\
\hline ecoli1 & 0.819 & 0.906 & 0.900 & 0.906 & 0.900 & 0.904 & 0.921 \\
\hline new-thyroid2 & 0.983 & 0.975 & 0.969 & 0.972 & 0.975 & 0.997 & 1.000 \\
\hline new-thyroid1 & 0.983 & 0.981 & 0.975 & 0.978 & 0.981 & 0.997 & 1.000 \\
\hline ecoli2 & 0.735 & 0.912 & 0.912 & 0.912 & 0.891 & 0.905 & 0.930 \\
\hline glass6 & 0.920 & 0.928 & 0.928 & 0.909 & 0.882 & 0.949 & 0.950 \\
\hline yeast3 & 0.630 & 0.902 & 0.908 & 0.904 & 0.903 & 0.900 & 0.927 \\
\hline yeast-2_vs_4 & 0.669 & 0.891 & 0.890 & 0.885 & 0.867 & 0.902 & 0.918 \\
\hline yeast-0-5-6-7-9_vs_4 & 0.500 & 0.774 & 0.791 & 0.785 & 0.786 & 0.796 & 0.817 \\
\hline vowel0 & 0.895 & 0.968 & 0.968 & 0.940 & 0.963 & 0.974 & 0.974 \\
\hline glass-0-1-6_vs_2 & 0.500 & 0.593 & 0.503 & 0.514 & 0.536 & 0.671 & 0.729 \\
\hline glass2 & 0.500 & 0.593 & 0.593 & 0.638 & 0.633 & 0.703 & 0.777 \\
\hline shuttle-c0-vs-c4 & 1.000 & 0.999 & 1.000 & 0.999 & 0.999 & 1.000 & 1.000 \\
\hline yeast-1_vs_7 & 0.500 & 0.748 & 0.753 & 0.759 & 0.760 & 0.766 & 0.782 \\
\hline glass4 & 0.559 & 0.898 & 0.905 & 0.918 & 0.900 & 0.960 & 0.969 \\
\hline ecoli4 & 0.575 & 0.948 & 0.935 & 0.945 & 0.910 & 0.979 & 0.981 \\
\hline glass-0-1-6_vs_5 & 0.497 & 0.937 & 0.943 & 0.920 & 0.951 & 0.969 & 0.971 \\
\hline shuttle-c2-vs-c4 & 1.000 & 0.975 & 0.996 & 0.910 & 0.980 & 1.000 & 1.000 \\
\hline yeast-1-4-5-8_vs_7 & 0.500 & 0.634 & 0.627 & 0.631 & 0.615 & 0.689 & 0.712 \\
\hline glass5 & 0.500 & 0.939 & 0.944 & 0.922 & 0.946 & 0.968 & 0.967 \\
\hline yeast-2_vs_8 & 0.774 & 0.761 & 0.763 & 0.742 & 0.735 & 0.842 & 0.830 \\
\hline yeast 4 & 0.500 & 0.823 & 0.815 & 0.821 & 0.822 & 0.771 & 0.763 \\
\hline yeast-1-2-8-9_vs_7 & 0.500 & 0.698 & 0.699 & 0.720 & 0.711 & 0.756 & 0.753 \\
\hline yeast5 & 0.500 & 0.963 & 0.962 & 0.960 & 0.961 & 0.965 & 0.975 \\
\hline ecoli-0-1-3-7_vs_2-6 & 0.850 & 0.842 & 0.851 & 0.822 & 0.790 & 0.967 & 0.970 \\
\hline yeast6 & 0.500 & 0.886 & 0.873 & 0.887 & 0.862 & 0.874 & 0.883 \\
\hline
\end{tabular}

\section{Conflict of Interests}

The authors declare that there is no conflict of interests regarding the publication of this paper.

\section{Acknowledgments}

This research was partially supported by the Natural Science Foundation of China under Grants nos. 61170020, 61402311, and 61440053, Jiangsu Province Colleges and Universities Natural Science Research Project under Grant no. 13KJB520021, Jiangsu Province Technology Innovation
Fund Project for Science and Technology Enterprises under Grant no. BC2013124, 2013 Suzhou Municipal Special Fund Project for Speeding up the Information Construction, the US National Science Foundation (IIS-1115417), and Jiangsu Province Postgraduate Cultivation and Innovation Project under Grant no. ZY32001814.

\section{References}

[1] N. V. Chawla, N. Japkowicz, and A. Kotcz, "Special issue on learning from imbalanced data sets," ACM SIGKDD Explorations Newsletter, vol. 6, no. 1, pp. 1-6, 2004. 
[2] H. He and E. A. Garcia, "Learning from imbalanced data," IEEE Transactions on Knowledge and Data Engineering, vol. 21, no. 9, pp. 1263-1284, 2009.

[3] C. Elkan, "The foundations of cost-sensitive learning," in Proceedings of the 17th International Joint Conference on Artificial Intelligence (IJCAI '01), pp. 973-978, August 2001.

[4] N. V. Chawla, K. W. Bowyer, L. O. Hall, and W. P. Kegelmeyer, "SMOTE: synthetic minority over-sampling technique," Journal of Artificial Intelligence Research, vol. 16, pp. 321-357, 2002.

[5] G. E. A. P. A. Batista, R. C. Prati, and M. C. Monard, "A study of the behaviour of several methods for balancing machine learning training data," SIGKDD Explorations, vol. 6, no. 1, pp. 20-29, 2004.

[6] D. L. Wilson, "Asymptotic properties of nearest neighbor rules using edited data," IEEE Transactions on Systems Man and Cybernetics, vol. 2, no. 3, pp. 408-421, 1972.

[7] H. Han, W. Y. Wang, and B. H. Mao, "Borderline-SMOTE: a new over-sampling method in imbalanced data sets learning," in Advances in Intelligent Computing: International Conference on Intelligent Computing, ICIC 2005, Hefei, China, August 23-26, 2005, Proceedings, Part I, vol. 3644 of Lecture Notes in Computer Science, pp. 878-887, Springer, Berlin, Germany, 2005.

[8] C. Bunkhumpornpat, K. Sinapiromsaran, and C. Lursinsap, "Safe-level-SMOTE: safe-level-synthetic minority oversampling technique for handling the class imbalanced problem," in Proceedings of the 13th Pacific-Asia Conference on Advances in Knowledge Discovery and Data Mining (PAKDD '09), pp. 475482, Bangkok, Thailand, April 2009.

[9] E. Ramentol, Y. Caballero, R. Bello, and F. Herrera, "SMOTE$\mathrm{RS} B *$ : a hybrid preprocessing approach based on oversampling and undersampling for high imbalanced data-sets using SMOTE and rough sets theory," Knowledge and Information Systems, vol. 33, no. 2, pp. 245-265, 2012.

[10] L. N. D. Castro and F. J. V. Zuben, "aiNet: an artificial immune network for data analysis," in Data Mining: A Heuristic Approach, H. A. Abbass, R. A. Sarker, and C. S. Newton, Eds., chapter 12, pp. 231-259, Idea Group Publishing, New York, NY, USA, 2001.

[11] R. Batuwita and V. Palade, "Efficient resampling methods for training support vector machines with imbalanced datasets," in Proceedings of the International Joint Conference on Neural Networks (IJCNN '10), pp. 1-8, IEEE, Barcelona, Spain, July 2010.

[12] A. Fernández, M. J. del Jesus, and F. Herrera, "On the 2tuples based genetic tuning performance for fuzzy rule based classification systems in imbalanced data-sets," Information Sciences, vol. 180, no. 8, pp. 1268-1291, 2010.

[13] A. Fernández, S. García, M. J. D. Jesus, and F. Herrera, "A study of the behaviour of linguistic fuzzy rule based classification systems in the framework of imbalanced data-sets," Fuzzy Sets and Systems, vol. 159, no. 18, pp. 2378-2398, 2008.

[14] V. López, A. Fernández, S. García, V. Palade, and F. Herrera, "An insight into classification with imbalanced data: empirical results and current trends on using data intrinsic characteristics," Information Sciences, vol. 250, pp. 113-141, 2013.

[15] T. Jo and N. Japkowicz, "Class imbalances versus small disjuncts," ACM SIGKDD Explorations Newsletter, vol. 6, no. 1, pp. 40-49, 2004

[16] N. K. Jerne, "Towards a network theory of the immune system," Annales d'immunologie, vol. 125C, no. 1-2, pp. 373-389, 1974.

[17] F. M. Burnet, "A modification of Jerne's theory of antibody production using the concept of clonal selection," CA: A Cancer Journal for Clinicians, vol. 26, no. 2, pp. 119-121, 1976.
[18] L. N. de Castro and F. J. von Zuben, "Learning and optimization using the clonal selection principle," IEEE Transactions on Evolutionary Computation, vol. 6, no. 3, pp. 239-251, 2002.

[19] J. Alcalá-Fdez, L. Sánchez, S. García et al., "KEEL: a software tool to assess evolutionary algorithms for data mining problems," Soft Computing, vol. 13, no. 3, pp. 307-318, 2009.

[20] G. J. McLachlan, Discriminant Analysis and Statistical Pattern Recognition, John Wiley \& Sons, New York, NY, USA, 2004.

[21] J. R. Quinlan, C4.5: Programs for Machine Learning, Morgan Kauffman, 1993.

[22] C. Cortes and V. Vapnik, "Support-vector networks," Machine Learning, vol. 20, no. 3, pp. 273-297, 1995.

[23] J. Alcalá-Fdez, A. Fernández, J. Luengo et al., "KEEL datamining software tool: data set repository, integration of algorithms and experimental analysis framework," Journal of Multiple-Valued Logic and Soft Computing, vol. 17, no. 2-3, pp. 255-287, 2011.

[24] A. P. Bradley, "The use of the area under the ROC curve in the evaluation of machine learning algorithms," Pattern Recognition, vol. 30, no. 7, pp. 1145-1159, 1997.

[25] J. Huang and C. X. Ling, "Using AUC and accuracy in evaluating learning algorithms," IEEE Transactions on Knowledge and Data Engineering, vol. 17, no. 3, pp. 299-310, 2005.

[26] J. G. Moreno-Torres and F. Herrera, "A preliminary study on overlapping and data fracture in imbalanced domains by means of genetic programming-based feature extraction," in Proceedings of the 10th International Conference on Intelligent Systems Design and Applications (ISDA '10), pp. 501-506, December 2010.

[27] J. G. Moreno-Torres, T. Raeder, R. Alaiz-Rodríguez, N. V. Chawla, and F. Herrera, "A unifying view on dataset shift in classification," Pattern Recognition, vol. 45, no. 1, pp. 521-530, 2012.

[28] H. He, Y. Bai, E. A. Garcia, and S. Li, "ADASYN: adaptive synthetic sampling approach for imbalanced learning," in Proceedings of the International Joint Conference on Neural Networks (IJCNN '08), pp. 1322-1328, June 2008.

[29] H. Han, W.-Y. Wang, and B.-H. Mao, "Borderline-SMOTE: a new over-sampling method in imbalanced data sets learning," in Proceedings of the International Conference on Intelligent Computing (ICIC '05), vol. 3644 of Lecture Notes in Computer Science, pp. 878-887, August 2005.

[30] J. P. Shaffer, "Modified sequentially rejective multiple test procedures," Journal of the American Statistical Association, vol. 81, no. 395, pp. 826-831, 1986. 

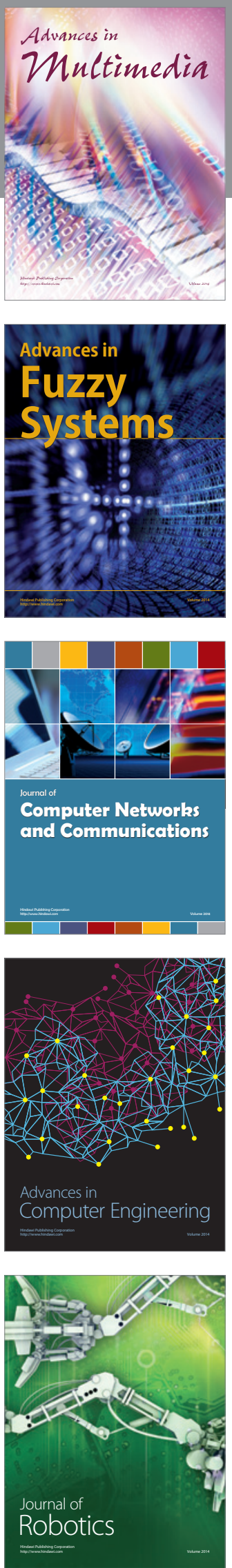

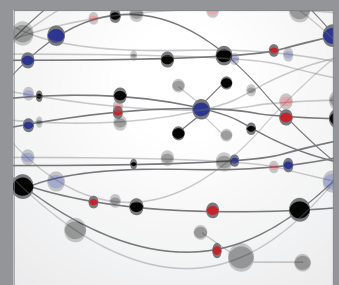

The Scientific World Journal
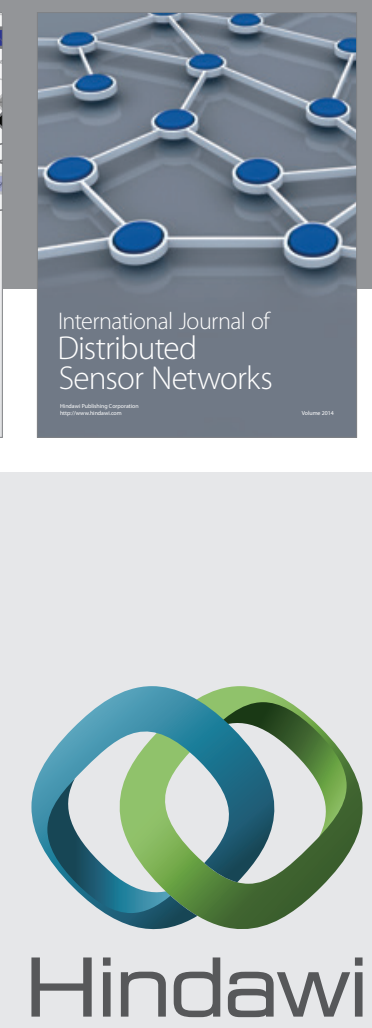

Submit your manuscripts at

http://www.hindawi.com
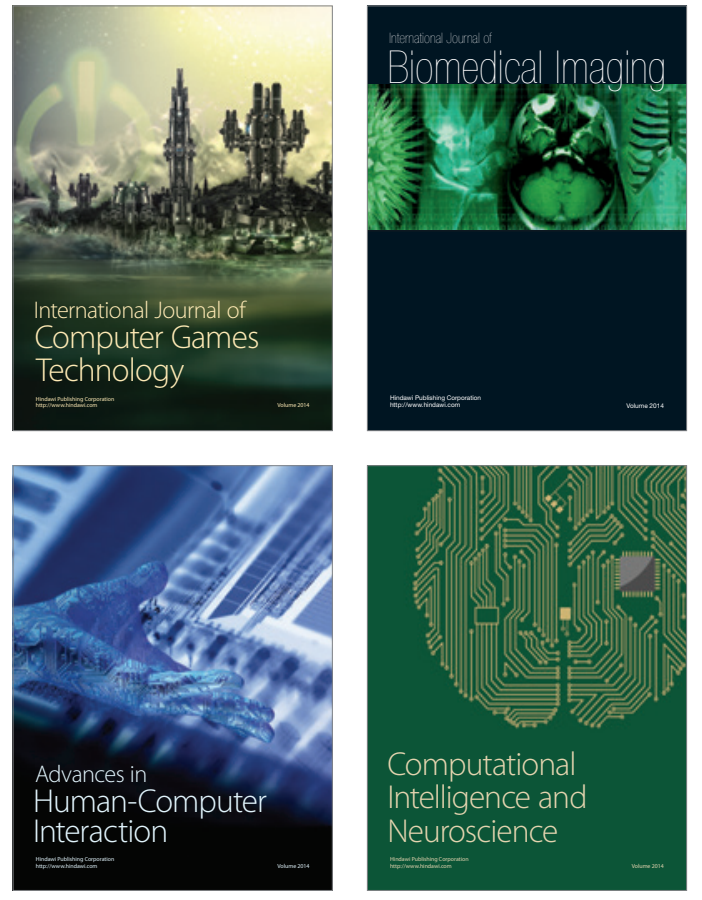
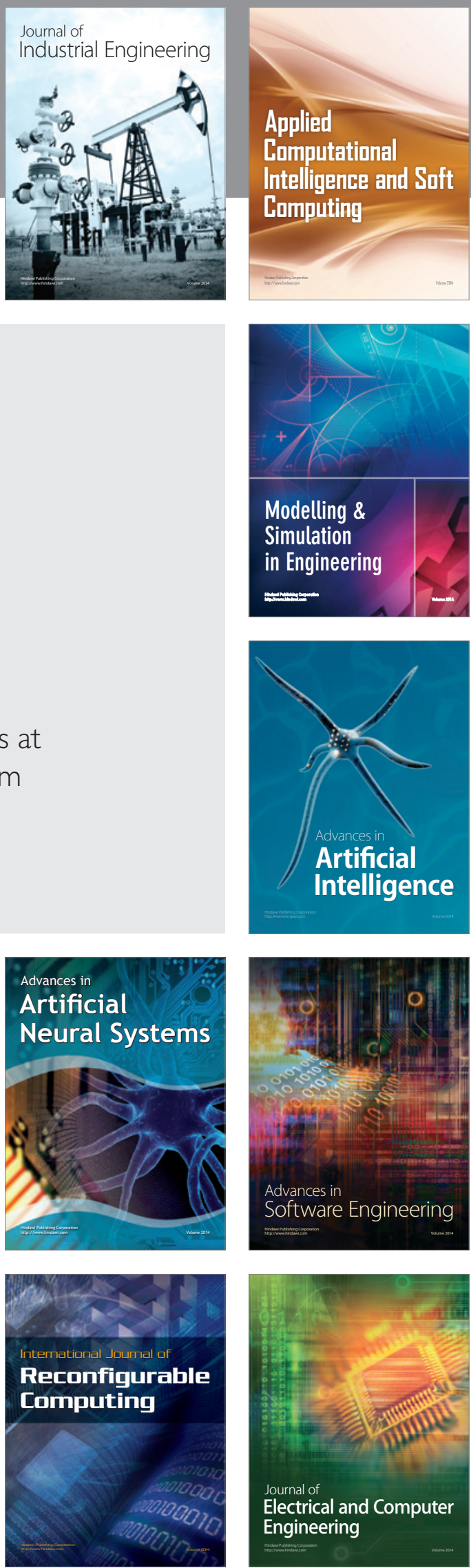